\title{
Mejorando Clima organizacional Y DE AUla, SATISFACCIÓN VITAL Y LABORAL ${ }^{\star}$
}

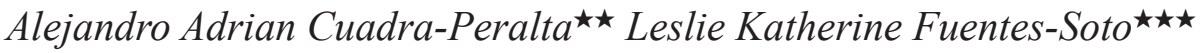 \\ Daniela Madueño-Soza $a^{\star \star \star \star}$ Constanza Beatriz Veloso-Besio $0^{\star \star \star \star \star}$

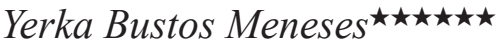

\begin{abstract}
RESUMEN
El siguiente trabajo es un intento de probar la efectividad de una intervención conjunta de psicología positiva con habilidades sociales en el contexto educativo. Para ello, se plantean cinco hipótesis referentes a las variables dependientes clima laboral, clima de aula, satisfacción vital y satisfacción laboral. Para probar lo anterior se utilizaron dos procedimientos cuasi experimentales. Los resultados muestran que la intervención basada en psicología positiva y habilidades sociales produce una significativa mejora en todas las variables concernientes a los profesores, a saber: satisfacción vital, satisfacción laboral y clima laboral. También produce un efecto positivo en el clima de aula de los alumnos, este efecto es más potente cuando se interviene en conjunto a los alumnos y a los profesores que cuando se trabaja sólo con los alumnos.

Palabras clave: habilidades sociales; psicología positiva; satisfacción vital; satisfacción laboral; clima laboral y clima de aula.

\section{IMPROVED CLIMATE ORGANIZATIONAL AND CLASSROOM, SATISFACTION WORK AND SATISFACCION WELL BEING}

\author{
Abstract \\ This paper is an attempt to test the effectiveness of a combined intervention of \\ positive psychology with social skills in the educational context. To do this, there

\footnotetext{
^ Se agradece el apoyo de Convenio de Desempeño MINEDUC - UTA

$\star \star$ Psicologo Universidad de Chile. Dr. en Psicología, Universidad Autonoma de Madrid. Académico Depto de Psicología. Universidad de Tarapacá, Arica- Chile. Dirección: 18 de Septiembre, 2222, Arica - Chile. E-mail: acuadra@uta.cl

$\star \star \star$ Psicologa Universidad de Tarapaca. Magister(c) Psicologia Social E-mail: lesliekfuentes@gmail.com

$\star \star \star \star$ Psicologa Universidad de TarapacaMagister(c) Psicologia Social E-mail:dani3la@live.cl

$\star \star \star \star \star$ Psicologa Universidad de Tarapaca. Magister Psicologia Social E-mail: veloso.constanza@gmail.com E-mail: yandreka25@gmail.com
} \\ $\star \star \star \star \star \star$ Psicologa Universidad de Tarapaca
}


Alejandro Adrian Cuadra-Peralta; Leslie Katherine Fuentes-Soto; Daniela Madueño-Soza;

Constanza Beatriz Veloso-Besio; Yerka Bustos Meneses

are five hypotheses concerning the dependent variables of work environment, classroom atmosphere, life satisfaction and job satisfaction. To test the above two procedures were used quasi-experimental. The results show that interventions based on positive psychology and social skills produces a significant improvement in all variables related to teachers, namely, life satisfaction, job satisfaction and labor climate. It also produces a positive effect on classroom atmosphere for students; this effect is strongest when it acts in conjunction with students and teachers when working only with students.

keywords: social skills; positive psychology; life satisfaction; job satisfaction; working environment and classroom atmosphere.

El presente trabajo muestra el resultado de una intervención cuyo objetivo fue mejorar la satisfacción vital, la satisfacción laboral y el clima laboral en profesores y simultáneamente generar un mejor clima de aula en los alumnos. También se probó la efectividad del programa trabajando solo con los alumnos versus la intervención conjunta de alumnos y profesores. La intervención se fundamentó en el marco de la psicología positiva y en los programas de entrenamientos en habilidades sociales.

\section{Psicología Positiva}

Según Seligman y Csikszentmihalyi (2000) antes de la Segunda Guerra Mundial, la psicología tenía tres misiones: curar la enfermedad mental, hacer la vida de las personas más plena e identificar y alimentar el talento. Sin embargo, los autores consideran que la única que prosperó hasta nuestros días es el estudio y tratamiento de enfermedades mentales, dentro de lo cual se han hecho grandes avances. En tanto, las otras dos misiones, hacer mejor la vida de las personas y fomentar "genios", han sido completamente olvidadas. Desde ese entonces (VERA POSECK, 2004), la psicología sigue el mismo rumbo y como consecuencia se encuentran instaladas creencias erróneas del tipo que la ausencia de enfermedad, es suficiente para alcanzar la felicidad o un grado óptimo de funcionamiento.

En este contexto surge la Psicología Positiva, como heredera directa del estudio de la satisfacción vital y la psicología de la felicidad (ARGYLE, 1987), con el objetivo de investigar acerca de las fortalezas y virtudes humanas y los efectos que éstas tienen en la vida de las personas y en las sociedades en que viven (CUADRA; FLORENZANO, 2003; VERA POSECK, 2004). De acuerdo a lo anterior, la psicología positiva representaría un nuevo punto de vista desde el cual poder entender la salud mental, afectiva y social, complementando la psicología ya existente, con la finalidad de alcanzar una mejor calidad de vida y bienestar, razón por la cual se llega necesariamente a la conclusión de la pertinencia de incluir dicha variable en la implementación de futuras intervenciones. 


\section{SATiSfacción Vital}

Uno de los conceptos característicos del enfoque teórico de la Psicología Positiva es la Satisfacción Vital, concepto que puede estudiarse desde un punto de vista afectivo o emocional considerando el equilibrio entre afecto positivo y afecto negativo (DÍAZ MORALES; SÁNCHEZ-LOPEZ, 2001), o bien, puede considerarse desde el punto de vista cognitivo, entendida como la valoración que la persona hace de su vida en general o de aspectos parciales de ésta, por ejemplo: familia, salud, estudios, trabajo o amigos. Por ende, la satisfacción con la vida, se refiere al proceso en el que las personas evalúan la calidad de sus vidas de acuerdo a su propio criterio y de manera global (MARTÍNEZ, 2004).

En relación a esto, Moyano y Ramos (2007) refieren el concepto de calidad de vida (C.V.), el cual estaría compuesto por una dimensión objetiva y otra subjetiva. Su dimensión objetiva se refiere a la disponibilidad de bienes y servicios de cada quién, mientras la subjetiva se relaciona con la valoración de la propia vida. Algunos autores plantean que la calidad de vida incluye al bienestar como componente subjetivo del concepto (LIBERALESSO, 2002, GARCÍA-VINIEGRAS; GONZÁLEZ, 2000), el que a su vez estaría conformado por aspectos cognitivos y afectivos. El componente cognitivo del bienestar sería la satisfacción vital, ya sea de manera global o por dominios específicos, y el componente afectivo se refiere a la presencia de sentimiento positivos, lo que algunos autores denominan felicidad (ARITA, 2005). En una dirección distinta a la precedente, hay quienes postulan a la felicidad como concepto mayor, inclusivo del bienestar y la satisfacción (CUADRA; FLORENZANO, 2003).

Un estudio realizados sobre la satisfacción con la vida (EMMONS; McCULLOUGH, 2003), demostró que practicar virtudes como la gratitud aumenta la satisfacción vital, argumentando que existe una fuerte relación entre bienestar subjetivo y fortalezas. Otro estudio relevante a considerar, se ha centrado más bien en variables internas o de personalidad, llegando a la deducción de que existe interacción entre personalidad y situación asociada a los niveles de satisfacción (DIENER; OISHI; LUCAS, 2003). Cuadra-Peralta et al. (2010a) lograron mejoras significativas en la disminución de sitomas depresivos y mejorar la satisfacción vital en pacientes con depresión severa, en una intervención basada en psicología positiva, mejorando la actitud hacia la vida y las relaciones interpersonales cercanas. En otras palabras, podría hablarse de dos maneras de entender la satisfacción: como el resultado de un conjunto de experiencias positivas (bottom-up) o como una disposición general de la personalidad (topdown), siendo la primera de éstas acepciones la que se considerara dentro del fundamento teórico del presente estudio.

\section{Satisfacción Laboral}

Entre los aspectos particulares constituyentes de la satisfacción vital, la cual anteriormente fue definida como la valoración positiva que la persona hace de su vida en general o de aspectos particulares de la misma, se encuentra la satisfacción con el trabajo (LIBERALESSO, 2002), existiendo evidencia de que la 
felicidad conduce a comportamientos que producen éxito en el trabajo, las relaciones sociales y la salud (LYUBOMIRSKY; KING; DIENER, 2005). Es así que la esta variable se entiende como un estado emocional positivo que resulta de la percepción subjetiva de la experiencia laboral (FERNÁNDEZ-RÍOS, 1999). Investigaciones sobre la conducta organizacional y su evaluación (CASTRO; SÁNCHEZ, 2000; MAÑAS et al., 2007; CUADRA-PERALTA; LEE MATURANA; SOSSA ROA, 2009) refieren que cada vez es más extendida en las organizaciones la idea de que la mejora en la satisfacción laboral de sus empleados no es sólo un medio para el logro de objetivos organizacionales, sino también, la posibilidad de que las personas disfruten de mejor salud mental y bienestar emocional. Lo anterior convierte a la satisfacción laboral en materia prioritaria de evaluación y mejora en cualquier ámbito organizacional, con mayor motivo lo será en el campo educativo, donde la labor que realizan los educadores tiene como materia prima a las personas en formación y como objetivo el guiar eficazmente su desarrollo hasta lograr que estén plena e íntegramente formadas. De hecho, se aprecia un creciente interés por el estudio de la satisfacción laboral de los docentes (DINHAM; SCOTT, 2000; GANZACH, 2003; METLER, 2002; PEARSON; MOARNAW, 2005; PRELIP, 2001; STEMPIEN; LOEB, 2002) en la medida que una sociedad avanzada debe velar porque sus educadores sean personas laboralmente satisfechas, ya que los beneficios que esto comporta, tanto para los individuos, como para las organizaciones devienen en calidad de la educación proporcionada y en la reputación de las instituciones educativas.

En suma, la satisfacción laboral ha sido definida como el resultado de varias actitudes que tiene un trabajador hacia su empleo y la vida en general. Si el trabajador tiene recursos suficientes, tanto personales como laborales, para afrontar las demandas, su estado psicológico será positivo con resultados como la satisfacción en su trabajo (SCHAUFELI; SALANOVA, 2002). Es así que, la relación de la Psicología Positiva y la satisfacción laboral, está determinada por las actitudes del individuo ante algo determinado, pudiendo resultar positivas o negativas según faciliten u obstruyan la manera que la persona tiene de afrontar su realidad en cada momento de su vida. Cabe mencionar que los buenos resultados no se logran sólo con buenas actitudes, además hay allí conocimientos, habilidades y experiencia, entre otros factores y es allí donde surge la necesidad del entrenamiento de determinadas habilidades, pero esto no será suficiente si la actitud es inadecuada ya que de esa forma, es difícil que las personas logren emprender un trabajo satisfactorio y menos disfrutar los éxitos alcanzados (SELIGMAN, 2002).

\section{Clima Organizacional}

La satisfacción laboral es una de las variables que interacciona de manera directa con el clima laboral (CUADRA-PERALTA; VELOSO-BESIO, 2007, 2010) abarcándola y complementándola, por esta razón dicha variable es incluida en el presente estudio. En esta línea y en relación al ámbito educacional, Martín Bris (1999) identifica cuatro percepciones que pueden ser consideradas como posturas teóricas de partida para analizar el concepto de clima: entendido como agente de presión ambiental percibido por los alumnos o los estudiantes; como 
función de las características típicas de los participantes; como función de las percepciones y actitudes de los profesores; o como calidad de vida en el centro educacional. Es así que las instituciones educativas pueden considerarse organizaciones sociales, puesto que, como lo señala Molina de Colmenares y Pérez de Maldonado (2006), están conformadas por un grupo de personas que ejercen determinadas funciones ordenadas por fines, objetivos y propósitos, dirigidos hacia la búsqueda de la eficiencia y la racionalidad. El clima organizacional representa, de alguna manera, un concepto global que integra todos los componentes organizacionales que se agrupan en los procesos y estructura organizacional.

Cabe señalar que diversas características del clima organizacional, tales como liderazgo, relación con los compañeros y estructura, han mostrado relaciones significativas con experiencias de estrés laboral y con las consecuencias negativas sobre la salud y el bienestar psicológico

Por último en el área del comportamiento organizacional y los recursos humanos, la Psicología Positiva aporta un modelo científico para poder evaluar y desarrollar objetivamente las fortalezas que resultan clave en la gestión efectiva del cambio y de personas. Permitiendo utilizar una nueva metodología de trabajo en la gestión y desarrollo de emociones positivas en el ámbito laboral, con un alto impacto positivo en el clima organizacional y la calidad de vida laboral. Lo anterior fomentaría el desarrollo de recursos intelectuales, físicos, psicológicos y sociales, que también son reforzados mediante el entrenamiento en Habilidades Sociales, posibilitando una mayor y mejor capacidad de resolución de problemas y toma de decisiones, caracterizada por un incremento en las capacidad de innovación y afrontamiento a las adversidades futuras, experimentando una mayor satisfacción con la vida laboral, personal y disfrutar con un mayor grado de felicidad auténtica el desempeño de su misión (FREDRICKSON, 2001; DIENER; SELIGMAN, 2004).

\section{Clima de Aula}

En consideración de que los objetivos del presente estudio se dirigen tanto hacia profesores como alumnos, es necesario referirse a otro aspecto del clima que surge dentro de las organizaciones educacionales además de los anteriormente descritos; el clima de aula. Este concepto tiene gran importancia a nivel educativo, pues se ha demostrado que la conducta del alumno varía en función de su percepción del clima social en la situación concreta en la que se desenvuelve. Son numerosos los autores que han realizado estudios y aportaciones sobre el tema y que corroboran esta afirmación (ASENSIO MUÑOZ; FERNÁNDEZ DÍAZ, 1991; ZABALZA, 1996; TRIANES, 2006; PÉREZ, 2007). De cualquier manera, es necesario tener en cuenta que el concepto de clima es amplio, siendo difícil comparar los resultados de investigaciones realizadas sobre el tema. Pese a ello, existen estudios que señalan una relación significativa entre la percepción del clima social escolar y el desarrollo emocional y social de alumnos y profesores (CORNEJO; REDONDO, 2001). Esta calidad de vida escolar estaría asociada a la sensación de bienestar general, confianza en las propias habilidades, creencia en la relevancia de lo que se aprende en la escuela, identificación con la misma, 
interacciones con pares y especialmente en las interacciones con los profesores. Es así que a la hora de promover un clima de relación humanizante es importante considerar que al realizar afirmaciones como "los alumnos son (o pueden ser) violentos o competitivos", en realidad no se están sino confirmando ideales y modos de enfrentarse a las situaciones cotidianas que les están siendo inculcados a través de las redes de socialización en las que se mueven (TORRES, 2001). El clima de aula no sólo puede describirse, sino modificarse, favoreciendo la cohesión social entre sus componentes. Para ello, es preciso dotarse de herramientas educativas que ayuden a su logro, sabiendo de la enorme dificultad, complejidad y diversidad de variables que están de trasfondo.

En el contexto escolar, las relaciones sociales que los niños tienen con profesores y compañeros ejercen una poderosa influencia tanto en su interés y motivación por la escuela como en su ajuste personal y social. En este sentido se ha constatado que aquellos alumnos que perciben un mayor grado de apoyo de sus profesores e iguales manifiestan también una mayor motivación e interés por las actividades escolares, son más proclives al cumplimiento de las normas que regulan el funcionamiento del aula, se implican más activamente en metas prosociales y su autoestima es más positiva (JESÚS CAVA; MUSITU, 2001). Un buen clima no se asocia de manera exclusiva con la disciplina y la autoridad, sino que se concibe de una manera mucho más global, abarcando también condiciones organizativas y culturales del aula y del establecimiento. Así Freiberg (1999), entiende el establecimiento educacional como un organismo vivo en el sentido cultural y organizativo, donde el clima de aula es el reflejo de la salud de dicho organismo.

Un estudio local (CUADRA-PERALTA, A. et al., 2010b), mediante la implementación de un programa basado en Psicología Positiva e Inteligencia Emocional, señala que el ámbito educacional podría verse muy beneficiado con la implementación de Psicología Positiva y Habilidades Sociales. Además, no puede obviarse que el profesor, dentro de la dinámica escolar de la sala de clases, no sólo produce el proceso de enseñanza-aprendizaje, sino que por el hecho de existir una permanente interacción alumno-profesor se vuelve necesario también establecer un clima afectivo-social que propicie aprendizajes significativos (CASSASUS, 2003). Según señala este autor, se demostró que si se suman todos los factores psicológicos extra escuela con los materiales y recursos humanos, esta suma es inferior a la importancia que tiene el clima emocional logrado en el aula (UNESCO, 2002). En suma, el clima social del establecimiento educacional y el clima de aula están en continuo intercambio, lo que debería exigir un esfuerzo de análisis y reflexión constante para delimitar las actuaciones más adecuadas. La formación del profesorado para comprender y tomar decisiones en el centro educativo, y singularmente en el aula, es un aspecto esencial para un desarrollo coherente del proceso de enseñanza y aprendizaje para la transformación social. La concepción de la enseñanza no debería limitarse a una reflexión instructiva, sino abrirse a la comprensión y transformación de la realidad sociointeractiva que se genera en el aula (ROMERO; CABALLERO, 2008). Por tanto, las implicaciones de los resultados en las tareas diarias de los docentes y alumnos son fáciles 
de deducir, pues las emociones y las habilidades sociales relacionadas con su manejo afectan los procesos de aprendizaje, calidad de las relaciones sociales y el rendimiento tanto académico como laboral (BRACKETT; CARUSSO, 2007).

\section{Habilidades Sociales}

Finalmente, y en concordancia a lo anteriormente señalado, surge la incorporación de las habilidades sociales al programa de intervención desarrollado en este estudio, ya que el entrenamiento en habilidades sociales ha demostrado su capacidad para mejorar el clima organizacional y que es un elemento esencial de la satisfacción vital (CUADRA-PERALTA et al., 2007) y que su entrenamiento mejora el ambiente de clase (WATSON, 2007).

Teniendo a la base el modelo de aprendizaje social, conviene tener en cuenta que las habilidades sociales son aprendidas y recíprocas, lo cual significa que en el ámbito educacional, el estudiante puede aprender conductas adecuadas por imitación, modelado, observación y refuerzos positivos, lo cual implica para el educador ser ejemplo en cuanto a actitudes. Una persona con un buen nivel de desarrollo de habilidades sociales tendrá mejores posibilidades de insertarse en el mundo social, participar de él y mantener relaciones interpersonales satisfactorias y efectivas (TAPIA, 2004). Las habilidades sociales tienen correlación con la autoestima, en la autorregulación del comportamiento, las relaciones con los compañeros de clases y de trabajo y en el rendimiento académico, entre otros aspectos, tanto en la infancia como en la vida adulta (LACUNZA; GONZÁLEZ, 2009). Son un mecanismo que facilita la protección y promoción de la salud mental, al favorece la interacción con los demás, y por ende la aceptación de los otros, la frecuencia de los refuerzos sociales positivo (LACUNZA; GONZÁLEZ, 2009).

Las habilidades sociales están íntimamente ligadas con la psicología positiva. Una de las variables que mas correlaciona con la satisfacción vital es la calidad de las relaciones interpersonales. El desarrollo de las habilidades sociales tiene como eje principal mejorar dichas relaciones, por ende es una competencia de gran significación para llevar una vida plena y feliz (CUADRA-PERALTA, A. et al., 2012).

En concordancia a lo anteriormente señalado, surge la incorporación de las habilidades sociales al programa de intervención desarrollado en este estudio, ya que el entrenamiento en habilidades sociales ha demostrado su capacidad para mejorar el clima organizacional, la satisfacción vital (CUADRA-PERALTA et al., 2007) y que su entrenamiento mejora el ambiente de clase (WATSON, 2007).

Es así que un programa basado en el entrenamiento conjunto de habilidades sociales y psicología positiva tanto al profesorado como al alumnado ayudarían a prevenir o abordar los conflictos que se den en el aula ya que permitirían una mejor comunicación de las necesidades de las partes, mayor respeto y soluciones a los conflictos, evitando que éstos se enquisten y dañen las relaciones de convivencia entre profesor-profesor, profesor-alumno y alumno-alumno. Por consiguiente, acorde a todo lo mencionado anteriormente, el objetivo general de 
la presente investigación será la adecuación, implementación y evaluación de un programa de entrenamiento en Psicología Positiva y Habilidades Sociales en alumnos y profesores de colegios particulares subvencionados de la ciudad de Arica. En base a ellos, se plantean cinco hipótesis estadísticas a contrastar:

H1: El programa de intervención mejorará significativamente el clima de aula de los alumnos.

H2: Habrá un incremento aún mayor del clima de aula en aquel grupo donde se intervendrá tanto a alumnos como a profesores respecto al grupo donde el programa se aplicará sólo a los alumnos.

H3: El programa de intervención aumentará significativamente la satisfacción vital de los profesores intervenidos.

H4: El programa de intervención aumentará significativamente la satisfacción laboral de los profesores intervenidos.

H5: El programa de intervención mejorará significativamente el clima laboral de los profesores intervenidos.

\section{MÉTodo}

\section{Participantes}

De un universo correspondiente a alumnos y profesores de enseñanza básica de colegios particulares subvencionados de la ciudad de Arica, se tomó una muestra de 216 alumnos de enseñanza básica (entre $5^{\circ}$ y $8^{\circ}$ año) de dos colegios particulares subvencionados de la ciudad de Arica. 138 de ellos pertenecientes al Colegio Saucache, donde un $47,1 \%$ de ellos son mujeres y un $52,9 \%$ hombres de una edad promedio de 12,5 años (DS=1,61). A su vez, los 78 alumnos restantes corresponden al Colegio San Marcos, donde un 43,6\% de los alumnos son mujeres y un 56,4\% hombres, alcanzando una edad promedio de 13,3 años (DS= 1,01). Finalmente, se contó con un total de 32 profesores, también provenientes del Colegio San Marcos, de los cuales sólo 21 de ellos participaron en el programa de intervención, con un $71,4 \%$ de integrantes mujeres y $28,6 \%$ hombres, con una edad promedio de 37,43 (DS=7,87). El grupo control estuvo constituido por los 11 profesores restantes de los cuales $54,5 \%$ eran mujeres y $45,5 \%$ hombres, con una edad promedio de $37,73 \%$, (DS=11,51).

\section{Variables}

Variable independiente: Programa de intervención.

Variable dependiente: Clima de Aula, Satisfacción Vital, Satisfacción Laboral y Clima Organizacional. 


\section{Instrumentos}

Para medir el clima de aula en alumnos, se utilizó el Inventario de Opinión Escolar "Mi Clase" I.M.C. Este inventario se caracteriza por ser una medida de alta inferencia, constituyendo una versión simplificada del "Inventario de Ambiente de Aprendizaje" (IAA) diseñado por Anderson (1982). Fue adaptado por Ascorra y Cáceres (2000-2001) para ser utilizado en muestras chilenas. El Inventario posee la estructura de una escala dicotómica, con un diseño simple de respuesta tipo SI y NO. Algunos ejemplos de reactivos son: "A ciertos alumnos no les gusta lo que hacen otros alumnos", "Todos los alumnos de mi clase se llevan bien entre sí" y "En nuestra clase los alumnos se consideran amigos entre sí". El nivel de confiabilidad de sus dimensiones es aceptable, obteniéndose Coeficiente Alpha para la dimensión Dificultad de 0.73, para Cohesividad 0.74, para Satisfacción 0.84, para Fricción 0.76 y para Competencia 0.63. El IMC. Cabe mencionar que este instrumento permite establecer adecuadamente el clima de aula general, es decir, el contexto en que se generan relaciones de confianza y las percepciones de los alumnos con respecto al entorno en que se desarrolla la clase.

De igual forma, para la evaluación del la satisfacción vital en profesores se utilizó la Escala de Satisfacción Laboral General, desarrollada por Warr, Cook, y Wall, (1979), la cual se compone de 15 ítems valorados en una escala liker de 5 puntos, donde 1 representa "muy insatisfecho" y 5 "muy satisfecho". Algunos ejemplos de reactivos son: "Condiciones físicas del trabajo" y "La atención que se presta a la sugerencias que haces". La escala permite la obtención de tres puntuaciones, correspondientes a: Satisfacción general (Coeficiente alpha entre .85 y .88), Satisfacción extrínseca (Coeficiente alpha entre .79 y .85) y Satisfacción intrínseca (Coeficiente Alpha entre .74 y .78).

Para evaluar la satisfacción vital se usó la Escala de Satisfacción Vital. Desarrollada por Ed. Diener (SELIGMAN, 2003), la cual consta de 5 ítems con alternativas de respuesta múltiple que evalúan la satisfacción con la vida de manera general. Cada afirmación es medida en una escala Likert de 7 puntos, donde 1 se presenta como "fuertemente en desacuerdo" y 7 "fuertemente de acuerdo". Algunos ejemplos de reactivos son: "En la mayoría de los aspectos mi vida se acerca a mi ideal" y "Hasta ahora, he conseguido las cosas más importantes que quiero en la vida". Además se estimó el coeficiente de fiabilidad a través del indicador Alpha, lo que arrojó un resultado de .95 .

Finalmente, para medir el clima organizacional se utilizo el Cuestionario de Clima Organizacional. Desarrollado por Cuadra-Peralta y Veloso-Besio (2007), el cual posee 11 dimensiones compuestas por 71 reactivos, los cuales se responden mediante una escala Likert de 5 puntos, donde 1 corresponde a "completamente en desacuerdo" y 5 "completamente de acuerdo". Algunos ejemplos de reactivos son: "En el colegio hay muchas oportunidades para desarrollarse profesionalmente" y "Las personas que trabajan en este liceo se esfuerzan por hacer bien su trabajo". Dicho cuestionario puede trabajarse de dos maneras, ya sea, dimensión por dimensión, o mediante una evaluación general 
Alejandro Adrian Cuadra-Peralta; Leslie Katherine Fuentes-Soto; Daniela Madueño-Soza;

Constanza Beatriz Veloso-Besio; Yerka Bustos Meneses

del clima, esto debido a la clara existencia de un factor general de segundo orden entre las diferentes sub-dimensiones, las cuales explican un 76\% de la varianza, que se denomina "Clima General".

\section{Diseño y Procedimiento}

La presente investigación se enmarcó en un diseño cuasi-experimental intergrupo pretest-postest, con variables intervalares. La muestra se dividió en tres grupos, dos experimentales a los cuales se les aplico el programa de intervención basado en Psicología Positiva y Habilidades Sociales: (G1) Solo alumnos, (G2) Alumnos y profesores, esto para probar la primera y segunda hipótesis, también se trabajó con un grupo control (G3), el cual estuvo conformado por profesores que no tuvieron ninguna intervención, éste último grupo fue utilizado en parte para probar las restantes hipótesis. Previo inicio de las sesiones, se aplicó el Inventario de Opinión Escolar a los alumnos y las Escalas de Satisfacción Vital, Satisfacción Laboral General y Cuestionario de Clima Organizacional a los profesores. El programa para ambos grupos constó de 13 sesiones con una programación semanal correspondiente a dos horas pedagógicas cada vez, correspondientes al horario de consejos de curso y consejo de profesores respectivamente. Cabe mencionar que la intervención se basó en un manual de Entrenamiento de Habilidades Sociales (CUADRA-PERALTA; FIGUEROA; PALZA, 2002), el cual fue adaptado y modificado para el caso de los profesores ya que la estructura de dicho manual está dirigida para alumnos. Conjuntamente, el programa de intervención para ambos grupos contuvo temáticas relacionadas con Psicología Positiva. Al finalizar la intervención, se evaluó nuevamente a los participantes mediante los mismos instrumentos anteriores.

Las actividades realizadas fueron las siguientes:

\begin{tabular}{|c|l|}
\hline $\begin{array}{c}\mathrm{N}^{\circ} \text { de } \\
\text { sesión }\end{array}$ & \multicolumn{1}{|c|}{ Actividad realizada } \\
\hline 1 & $\begin{array}{l}\text {-Evaluación pre - intervención - Actividad de presentación (Pre- } \\
\text { Capacitación). }\end{array}$ \\
\hline 2 & $\begin{array}{l}\text {-Presentación de objetivos y estructura del programa de interven- } \\
\text { ción. } \\
\text {-Relevancia y aplicabilidad de las Habilidades Sociales y la Psico- } \\
\text { logía Positiva en la vida diaria (dentro y fuera del trabajo). }\end{array}$ \\
\hline 3 & $\begin{array}{l}\text {-Entrenamiento en Habilidades Sociales: Importancia del estableci- } \\
\text { miento de relaciones sociales. } \\
\text {-Entrenamiento en Psicología Positiva: Aplicación del Cuestionario } \\
\text { de Fortalezas Personales (VIAS). }\end{array}$ \\
\hline 4 & $\begin{array}{l}\text {-Entrenamiento en Habilidades Sociales: Iniciar y mantener con- } \\
\text { versaciones. } \\
\text {-Entrenamiento en Psicología Positiva: Entrega de resultados del } \\
\text { Cuestionario de Fortalezas Personales (VIAS); Revisión y análisis } \\
\text { de bitácoras relacionadas con el uso de las fortalezas personales. }\end{array}$ \\
\hline
\end{tabular}




\begin{tabular}{|c|c|}
\hline $\begin{array}{l}\mathrm{N}^{\circ} \text { de } \\
\text { sesión }\end{array}$ & Actividad realizada \\
\hline 5 & $\begin{array}{l}\text {-Entrenamiento en Habilidades Sociales: Expresión de opiniones } \\
\text { personales. } \\
\text {-Entrenamiento en Psicología Positiva: Concepto de Optimismo. }\end{array}$ \\
\hline 6 & $\begin{array}{l}\text {-Entrenamiento en Habilidades Sociales: ¿Qué es la Asertividad?. } \\
\text {-Entrenamiento en Psicología Positiva: Revisión de bitácoras rela- } \\
\text { cionadas con el Optimismo. }\end{array}$ \\
\hline 7 & $\begin{array}{l}\text {-Entrenamiento en Habilidades Sociales: Expresión de agrado y } \\
\text { afecto. } \\
\text {-Entrenamiento en Psicología Positiva: Actividad: Recuerdo de } \\
\text { momentos felices. }\end{array}$ \\
\hline 8 & $\begin{array}{l}\text {-Entrenamiento en Habilidades Sociales: Hacer y rechazar peticio- } \\
\text { nes. } \\
\text {-Entrenamiento en Psicología Positiva: Revisión y análisis de bitá- } \\
\text { coras relacionadas con la instrucción de prestar atención a eventos } \\
\text { positivos del diario vivir. }\end{array}$ \\
\hline 9 & $\begin{array}{l}\text {-Entrenamiento en Habilidades Sociales: Defensa de los derechos. } \\
\text {-Entrenamiento en Psicología Positiva: Humor positivo. }\end{array}$ \\
\hline 10 & $\begin{array}{l}\text {-Entrenamiento en Habilidades Sociales: Hacer y recibir cumpli- } \\
\text { dos. } \\
\text {-Entrenamiento en Psicología Positiva: Expresión de Gratitud. }\end{array}$ \\
\hline 11 & $\begin{array}{l}\text {-Entrenamiento en Habilidades Sociales: Expresión de molestia, } \\
\text { desagrado y enfado y Afrontamiento de críticas. } \\
\text {-Entrenamiento en Psicología Positiva: ¿Qué es el flow (fluir)?. }\end{array}$ \\
\hline 12 & $\begin{array}{l}\text {-Entrenamiento en Habilidades Sociales y en Psicología Positiva: } \\
\text { Técnicas de control de agresividad y emociones negativas }\end{array}$ \\
\hline 13 & -Actividad de cierre \\
\hline
\end{tabular}

\section{Resultados}

A lo largo de este apartado se expondrán los resultados obtenidos de los análisis estadísticos realizados, con el fin de poner a prueba cada una de las hipótesis planteadas.

Tabla N1. Comparación de medias para Clima de Aula en alumnos Pre y Post intervención. Ambos grupos: Grupo solo alumnos y Grupo alumnos y profesores

\begin{tabular}{lccccccccc} 
& \multicolumn{9}{c}{ PRE } \\
\cline { 2 - 9 } & $\mathrm{N}$ & Media & $\begin{array}{c}\text { Desviación } \\
\text { Típ. }\end{array}$ & $\mathrm{N}$ & Media & $\begin{array}{c}\text { Desviación } \\
\text { Típ. }\end{array}$ & $\begin{array}{c}\text { Dif. de } \\
\text { Media }\end{array}$ & t & Sig. \\
\hline $\begin{array}{l}\text { CLIMA } \\
\text { DE AULA }\end{array}$ & 216 & 4,40 & 2,56 & 216 & 7,76 & 2,02 & 3,36 & 15,16 &, $000^{* *}$ \\
\hline
\end{tabular}

$* *$ Significativo al 0,05

Nota: Pre: datos antes del programa de Intervención Post: datos después del programa de Intervención 
La tabla N1 proporciona las medias pre y post intervención de la variable dependiente Clima de Aula en alumnos, respecto a la cual es posible analizar la primera hipótesis de éste estudio: "El programa de intervención mejorará significativamente el clima de aula de los alumnos", donde la media de la condición post-intervención $(7,76)$ es mayor a la obtenida pre intervención $(4,40)$, obteniéndose una diferencia de medias de 3,36 $[\mathrm{t}(215)=15,16 ; \mathrm{p}<0,05]$. Para efecto de probar la $\mathrm{H} 2$, se evaluó si había diferencias significativas según la condición de intervención, a saber sólo los alumnos versus alumnos en conjunto con los profesores. A continuación la tabla N2 indica los valores para la comprobación de dicha hipótesis.

Tabla N2. Diferencia de medias en Clima de Aula Pre y Post intervención por condición

\begin{tabular}{|c|c|c|c|c|c|c|c|c|c|c|c|c|}
\hline & \multicolumn{6}{|c|}{ PRE } & \multicolumn{6}{|c|}{ POST } \\
\hline & \multicolumn{2}{|c|}{$\begin{array}{l}\text { Sólo Alumnos } \\
\qquad(\mathrm{N}=138)\end{array}$} & \multicolumn{2}{|c|}{$\begin{array}{l}\text { Alumno s+prof. } \\
\qquad(\mathrm{N}=78)\end{array}$} & \multirow[b]{2}{*}{$\mathrm{t}$} & \multirow[b]{2}{*}{ Sig. } & \multicolumn{2}{|c|}{$\begin{array}{c}\text { Sólo } \\
\text { Alumnos } \\
(\mathrm{N}=138)\end{array}$} & \multicolumn{2}{|c|}{$\begin{array}{l}\text { Alumnos+prof. } \\
(\mathrm{N}=78)\end{array}$} & \multirow[b]{2}{*}{$\mathrm{t}$} & \multirow[b]{2}{*}{ Sig. } \\
\hline & Media & $\begin{array}{l}\text { Des. } \\
\text { Típ. }\end{array}$ & Media & $\begin{array}{l}\text { Des. } \\
\text { Típ. }\end{array}$ & & & Media & $\begin{array}{l}\text { Des. } \\
\text { Típ. }\end{array}$ & Media & $\begin{array}{l}\text { Des. } \\
\text { Típ. }\end{array}$ & & \\
\hline $\begin{array}{l}\text { CLIMA } \\
\text { DE AULA }\end{array}$ & 5,09 & 2,69 & 3,19 & 1,74 & 5,58 &, $00^{* *}$ & 7,41 & 2,11 & 8,40 & 1,67 & 3,56 &, $00^{* *}$ \\
\hline
\end{tabular}

**Significativo al 0,05

Nota: Pre: datos antes del programa de Intervención Post: datos después del programa de Intervención

Como se mencionó anteriormente, esta tabla proporciona información respecto a la segunda hipótesis planteada: "Habrá un incremento aún mayor del clima de aula en aquel grupo donde se aplicó el programa de intervención tanto a alumnos como a profesores", observándose una diferencia significativa entre las medidas post-intervención de ambos grupos $(7,41$ y 8,40) respectivamente, con una significativa diferencia de medias de 0,99 y $[\mathrm{t}(215)=3,563 ; \mathrm{p}<0,05]$. Cabe señalar que al igual que en las medias post intervención, existieron diferencias significativas en las medias pre intervención entre los grupos experimentales, observándose valores iníciales mayores en G1 compuesto solo por alumnos $(5,09)$ sobre la medias del G2 compuesto por alumnos y profesores $(3,19)$, lo cual indica una diferencia de medias significativa de 1,89 [t(215) $=5,58 ; \mathrm{p}<0,05]$. 
Figura N1. Medias estimadas para Clima de Aula Pre y Post por condición experimental

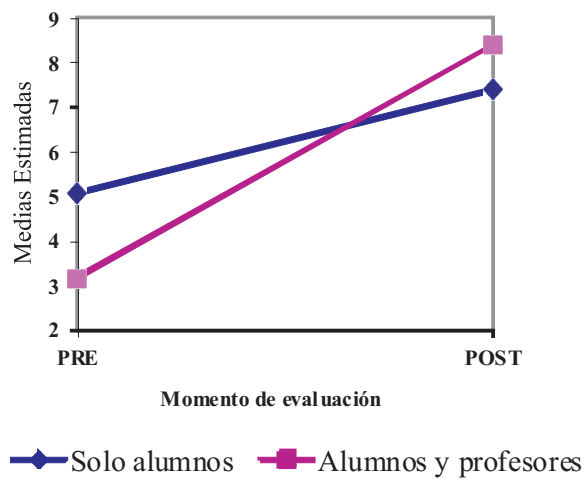

La figura N1 grafica las diferencias de medias obtenidas en Clima de Aula para ambos grupos (GI: sólo alumnos; G2: alumnos y profesores) en los dos momentos de evaluación (Pre y Post intervención), es decir, muestra la efectividad del programa de intervención en base al aumento significativo de la pendiente de G2 en la post intervención en relación a la media de G1 en el mismo momento de evaluación.

Tabla N3. Comparación de medias pre y post-intervención de las variables Satisfacción vital, Satisfacción laboral y Clima Laboral en el grupo experimental de profesores.

\begin{tabular}{|c|c|c|c|c|c|c|c|}
\hline & \multicolumn{7}{|c|}{$\begin{array}{c}\text { Intervención en Profesores } \\
\text { (Grupo Experimental) } \\
(\mathrm{N}=21)\end{array}$} \\
\hline & \multicolumn{2}{|c|}{ PRE } & \multicolumn{2}{|c|}{ POST } & \multirow[t]{2}{*}{$\begin{array}{l}\text { Dif. de } \\
\text { Medias }\end{array}$} & \multirow[t]{2}{*}{$\mathrm{t}$} & \multirow[t]{2}{*}{ Sig. } \\
\hline & Media & Desv. Típ. & Media & $\begin{array}{l}\text { Desv. } \\
\text { Típ. }\end{array}$ & & & \\
\hline Satisfacción Vital & 20,71 & 5,24 & 27,52 & 4,59 & 6,81 & 4,10 & $.000^{* *}$ \\
\hline Satisfacción Laboral & 43,29 & 3,82 & 51,14 & 2,94 & 7,86 & 6,46 & .000 ** \\
\hline Clima Laboral & 2,34 & 20 & 3,19 & 27 &, 850 & 11,68 & $.000^{* *}$ \\
\hline
\end{tabular}

**Significativo al 0,05

Nota: Pre: datos antes del programa de Intervención Post: datos después del programa de Intervención

Referente a la tercera hipótesis "El programa de intervención aumentará la satisfacción vital de los profesores", en la tabla N3 se observa un aumento significativo de los medias pre y post del grupo de profesores intervenidos, siendo estas de $(20,71)$ y $(27,52)$ correspondientemente, con una diferencia de medias de $6,81[\mathrm{t}(20)=4,097 ; \mathrm{p}<0,05]$. De igual forma, respecto a la cuarta hipótesis "El programa de intervención aumentará la satisfacción laboral de los profesores", la tabla indica un aumento significativo de las medias, siendo éstas $(43,29)$ pre 
intervención y $(51,14)$ post intervención, con una diferencia de medias de 7,86 $[\mathrm{t}(20)=6,455 ; \mathrm{p}<0,05]$. Finalmente, respecto a la quinta hipótesis planteada "El programa de intervención mejorará el clima laboral de los profesores" se observa que la media de la condición post-intervención $(3,188)$ fue significativamente mayor que la media de la condición pre-intervención $(2,338)$, con una diferencia de medias de, $850[\mathrm{t}(20)=11,67 ; \mathrm{p}<0,05]$.

Tabla N4. Comparación de medias post intervención para las variables, Satisfacción vital, Satisfacción laboral y Clima Laboral de los grupos control y experimental de profesores

\begin{tabular}{lccccccc}
\hline & \multicolumn{2}{c}{$\begin{array}{c}\text { Grupo control } \\
\text { (N=11) } \\
\text { Post }\end{array}$} & \multicolumn{2}{c}{$\begin{array}{c}\text { Grupo Experimental } \\
\text { (N=21) } \\
\text { Post }\end{array}$} \\
\cline { 2 - 6 } & Media & D.S. & Media & D.S. & $\begin{array}{c}\text { Dif. de } \\
\text { Medias }\end{array}$ & t & Sig. \\
\hline Satisfacción Vital & 19,91 & 4,46 & 27,52 & 5,24 & 7,61 & 4,31 &, $000^{* *}$ \\
\hline Satisfacción Laboral & 41,73 & 2,97 & 51,14 & 4,32 & 9,42 & 7,24 &, $000^{* *}$ \\
\hline Clima Laboral & 2,29 &, 20 & 3,19 &, 268 &, 90 & 10,62 &, $000^{* *}$ \\
\hline
\end{tabular}

**Significativo al 0,05

Nota: Post: datos después del programa de Intervención

Es conveniente señalar que no había diferencias significativas antes de la intervención entre los gropos control y experimental en ninguna de las tres variables analizada Sin embargo, la situación es diferente respecto a las medias post intervención, donde si existen diferencias significativas entre ambos grupos, siendo sus medias de $(27,52)$ y $(19,91)[\mathrm{t}(30)=4,31 ; \mathrm{p}<0,05]$ para satisfacción vital, $(51,14)$ y $(41,73)[\mathrm{t}(30)=7,24 ; \mathrm{p}<0,05]$ para satisfacción laboral y $(3,18)$ y $(2,20)$ $[\mathrm{t}(30)=10,62 ; \mathrm{p}<0,05]$ para clima laboral.

En concordancia con lo antes planteado, la figura N2 grafica el efecto del programa de intervención sobre ambos grupos de participantes, es decir, muestra las medias pre y post intervención del grupo experimental y control simultáneamente para cada una de las variables estudiadas en la presente investigación. 
Figura N2. Gráfico de medias estimadas para Satisfacción Vital, Laboral y Clima Organizacional Pre y Post por grupo de intervención
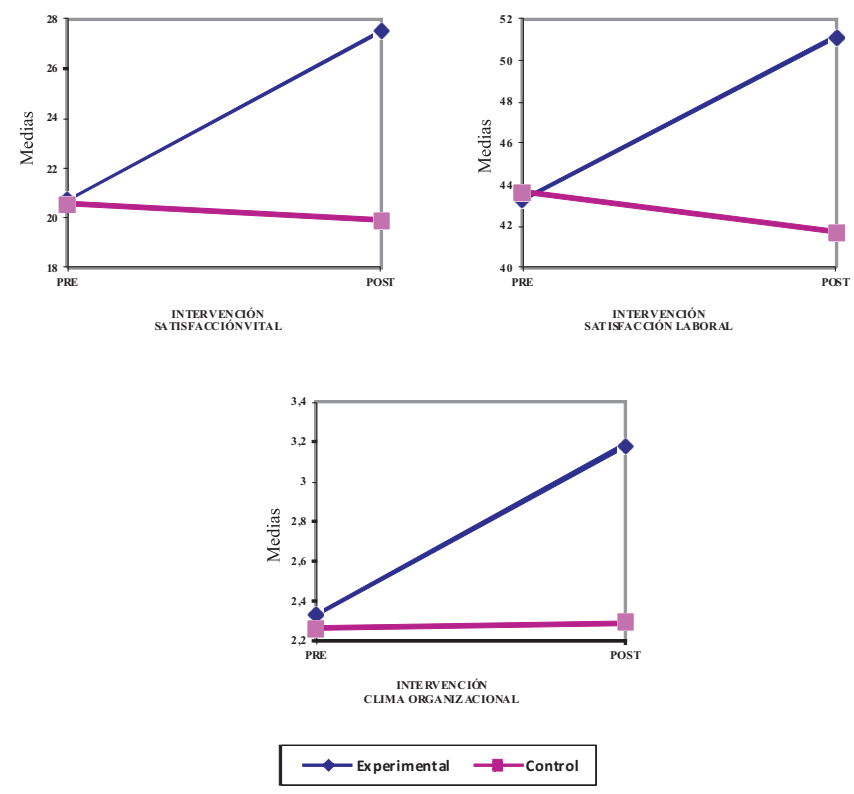

\section{Discusión}

El objetivo general consistió en evaluar el efecto de un programa de intervención basado en Psicología Positiva y Habilidades Sociales sobre: clima de aula, satisfacción vital, satisfacción laboral y clima organizacional. Los resultados obtenidos evidencian efectividad del programa, confirmándose cada una de las hipótesis planteadas.

En el caso de la primera hipótesis ("El programa de intervención mejorará significativamente el clima de aula de los alumnos"), ésta es confirmada al observarse un incremento significativo en las medias post-intervención, reafirmando aquellos estudios que señalan una relación significativa entre la percepción del clima social escolar y el desarrollo emocional y social de alumnos y profesores (CORNEJO; REDONDO, 2001).

Respecto a la segunda hipótesis propuesta en este estudio ("Habrá un incremento aún mayor del clima de aula en aquel grupo donde se aplicó el programa de intervención tanto a alumnos como a docentes"), ésta es corroborada mediante una diferencia significativa en las medias post-intervención de ambos grupos. El grupo intervenido tanto a alumnos como a profesores (G2) obtuvo mejores resultados post-intervención que el grupo donde sólo se intervino a los alumnos (G1), como se puede apreciar en la tabla N2, aun cuando éste reportaba medias pre-intervención menores en el clima de aula. Por ende el impacto del programa de intervención sobre G2 sería más significativo respecto a G1. 
En síntesis, es posible referir que una intervención basada en Habilidades Sociales y Psicología positiva efectivamente incrementa y mejora el clima de aula en estudiantes, siendo este acrecentamiento aun mejor cuando la intervención es dirigida simultáneamente a los profesores de dichos alumnos, comprobando así la influencia que tiene el comportamiento y/o actitudes de los docentes sobre la percepción que tienen los alumnos del entorno que los rodea dentro del establecimiento educacional. Entonces, si se considera que el clima de aula no sólo puede describirse, sino además modificarse favoreciendo la cohesión social entre sus componentes, surge la necesidad de que tantos profesores como alumnos, se doten de las herramientas necesarias para el logro de este, entre las cuales, como se ha podido comprobar en este estudio, está la implementación de un programa basado en el entrenamiento de Habilidades Sociales y Psicología Positiva.

De igual forma, al confirmarse la tercera hipótesis ("El programa de intervención aumentará la satisfacción vital de los profesores"), se refuerza la idea de que los vínculos personales cercanos resultan ser una de las fuentes de mayor satisfacción, pues actúan como redes de apoyo y a la vez reafirma la correlación existente entre satisfacción vital y la práctica de virtudes, así como también de fortalezas (McCULLOUGH; PARGAMENT; THORESEN, 2000; EMMONS; McCULLOUGH, 2003).

Siguiendo en la misma línea, la ratificación de la cuarta hipótesis de la presente investigación ("El programa de intervención aumentará la satisfacción laboral de los profesores"), respalda el planteamiento del estudio realizado por Schaufeli y Salanova (2002), quienes postulan que si un trabajador tiene recursos tanto personales como laborales suficientes para afrontar las demandas, su estado psicológico será positivo, con resultados favorables en la satisfacción con su trabajo. Así mismo tal como señala (ROMERO; CABALLERO, 2008), el clima social del establecimiento educacional y el clima de aula están en continuo intercambio, lo que debería exigir un esfuerzo de análisis y reflexión constante para delimitar las actuaciones más adecuadas. Por ello la formación del profesorado para comprender y tomar decisiones en el centro educativo, y singularmente en el aula, es un aspecto esencial para un desarrollo coherente del proceso de enseñanza y aprendizaje para la transformación social.

En suma, si se considera que el clima laboral es una variable ampliamente estudiada como elemento importante a nivel de satisfacción de los docentes, era esperable que una intervención dirigida hacia la mejora de las relaciones interpersonales basado en la implementación de la Psicología Positiva y las Habilidades Sociales obtuviera efectos positivos sobre variables como satisfacción vital y laboral, pues como se planteó anteriormente, el tipo de relaciones interpersonales que mantengan y de la cohesión que se genere, dependerá, en parte, su nivel de satisfacción con la vida y por ende con el trabajo.

Con respecto a la última hipótesis ("El programa de intervención mejorará el clima laboral de los profesores"), ésta se confirma mediante los resultados obtenidos, siendo concordante con los planteamientos de Klein et al. (2001), según quienes la comunicación y las repetidas interacciones sociales entre miembros de 
la misma organización influencian puntos de vistas individuales y también pueden contribuir al desarrollo de significados y percepciones compartidas. Además, si se considera, que el clima laboral es una manifestación externa (percepción) de los sujetos respecto a las variables organizacionales, era de suponer que una intervención de este tipo influyera positivamente en la forma de percibir la organización por parte de los docentes. En este contexto, se convierte en prioridad que los docentes tengan una actitud positiva hacia su trabajo. Por último es pertinente mencionar que las medias pre-intervención de ambos grupos aluden a una igualdad de condiciones, es decir, no tienen diferencias significativas.

En definitiva, se concluye un impacto positivo del programa de intervención diseñado para profesores en base al entrenamiento en Habilidades Sociales y Psicología Positiva sobre las variables estudiadas (satisfacción vital, satisfacción laboral y clima organizacional) respecto a la no intervención, por ende, es posible señalar que la implementación del programa produce cambios favorables y significativos en profesores referente a las variables ya mencionadas, y que dichas variables no mejoran por si solas o por efecto alguna otra circunstancia como quedo demostrado en los resultados del grupo control o no intervenido.

Se ha analizado una problemática real y en continuo crecimiento exponencial, realizándose diligentemente, sin embargo existen aspectos que escapan a la líneas de acción, es por ello que se especifican aspectos que sería apropiado estudiar más a fondo en futuras investigaciones como el análisis del impacto del programa sobre otros niveles socioeconómicos incluyendo en la muestra colegios municipales y particulares pagados. De igual forma, si se consideró como punto fundamental la influencia del profesor sobre la percepción del clima de aula de los alumnos, sería importante que en otras investigaciones se considerara la posible influencia existente desde alumnos hacia profesores, es decir, hacer un análisis bidireccional del fenómeno.

Sin embargo, a pesar de las limitaciones antes expuestas, esta investigación constituye un modelo de intervención sencillo que puede aplicar programas de educación y evaluar la eficacia de los mismos. Se debe tener en cuenta que en nuestros centros educativos se realizan muchas intervenciones pero pocas veces se organizan actividades que busquen objetivos claros y concretos, y rara vez se evalúan los resultados obtenidos. Por otra parte, la investigación realizada puede constituir un punto de partida para investigaciones más amplias, en base a la aplicación de programas de intervención en la convivencia que han resultado eficaces. Se dispone de un gran número de investigaciones sobre el tema, pero desafortunadamente, la mayor parte de ellas se limitan a establecer un diagnóstico de la situación y a sugerir las propuestas de intervención, siendo escasas las investigaciones centradas en la confección de programas de intervención y aplicación de los mismos.

Se comenzó esta investigación planteando que las investigaciones empíricas realizadas hasta el momento, no habían incluido un programa de intervención dirigido en forma conjunta al alumnado y profesorado, como tampoco se había incorporado un entrenamiento en Psicología Positiva y Habilidades Sociales conjuntamente, por lo cual no se había podido llegar a conclusiones firmes relativas 
al clima de aula y la posible mutua influencia en la satisfacción vital, laboral y clima organizacional de los docentes. En consideración a esto, es posible plantear, que el presente estudio de alguna forma suple aquel vacío, siendo un aporte para la investigación en la psicología educacional y laboral.

\section{REFERENCIAS}

ANDERSON, C. The search of school climate. Review of Educational Research, [S.1.], v. 52, n. 3, p. 368-420, 1982.

ASCORRA, P.; CÁCERES, P. Evaluación de los aspectos psicométricos del inventario de clima de aula "Mi Clase". Revista Enfoques Educacionales, v. 3, n. 1, p. 117-134, 2001. Santiago de Chile: Universidad de Chile.

ARGYLE, M. La psicología de la felicidad. Madrid: Alianza, 1987.

ARITA, B. Satisfacción por la vida y teoría homeostática del bienestar, Psicología y Salud, [S.1.], v. 15, n. 1, p.121-126, 2005.

ASENSIO MUÑOZ, I.; FERNÁNDEZ DÍAZ, M. J. El clima de las Instituciones de Educación Superior. Revista Complutense de Educación, [S.1.], v. 2, n. 3, p.501-518, 1991.

BRACKETT, M.; CARUSSO, D. Emotionally literacy for educators. Cary, NC: SEL-media, 2007.

CASSASUS, J. La escuela y la (des)igualdad. Santiago: LOM, 2003.

CASTRO, A.; SÁNCHEZ, M. P. Objetivos de vida y satisfacción autopercibida en estudiantes universitarios. Psicothema, [S.1.], v. 12, n. 2, p. 87-92, 2000.

CORNEJO, R.; REDONDO, M. El clima escolar percibido por los alumnos de Enseñanza Media: una investigación en algunos liceos de la Región Metropolitana, Chile. Ultima Década, [S.1.], v. 9, n. 15, p. 11-52, 2001.

CUADRA-PERALTA, A. et al. Capacitación en liderazgo. CONGRESO CHILENO DE PSICOLOGÍA, 2., Santiago. Anais... Santiago de Chile: Escuelas de Psicología del Consorcio de Universidades del Estado de Chile, 3 al 6 de Octubre 2007. 
CUADRA-PERALTA, A. et al. Resultados de la psicoterapia positiva en pacientes con depresión. Terapia Psicológica, [S.1.], v. 28, n. 1, p. 127-134, 2010a. Disponible em: <http://www.scielo.cl/scielo.php?script=sci_arttext\&pid $=$ S0718-48082010000100012>. Consultado en 22 mayo 2011.

CUADRA-PERALTA, A. et al. Efecto de un programa de psicología positiva e inteligencia emocional sobre la satisfacción laboral y vital. Revista Salud \& Sociedad, [S.1.], v. 1, n. 2, 2010b. Disponible en: <http://www.saludysociedad.cl/ index.php/main/article/view/17/37>. Consultado en 22 mayo 2011.

CUADRA-PERALTA, A. et al. Impacto de una intervención en psicología positiva sobre síntomas depresivos y satisfacción vital en adultos mayores. Psicología: Reflexión y Crítica, [S.1.], v. 25, n. 4. 2012. Artículo aceptado para publicación.

CUADRA-PERALTA, A; VELOSO-BESIO, C. Liderazgo, clima y satisfacción laboral en las organizaciones. Universum, Talca, v. 22, n. 2, p. 40-56, 2007. Disponible en: <http://www.scielo.cl/scielo.php?script=sci_arttext\&pid=S0718$23762007000200004 \& \operatorname{lng}=e s \& n r m=i s o>$. Consultado en 22 mayo 2011.

CUADRA-PERALTA, A.; VELOSO-BESIO, C. Grado de supervisión como variable moderadora entre liderazgo y satisfacción, motivación y clima organizacional. Ingeniare. Rev. chil. Ing., [S.1.], v. 18, n. 1, p. 15-25, 2010. Disponible en: $<\mathrm{http} / / \mathrm{www}$.scielo.cl/scielo.php?script=sci_arttext\&pid=S07183 3052010000100003\&lng=es\&nrm=iso>. Consultado en 22 mayo 2011.

CUADRA-PERALTA, A; LEE MATURANA, S; SOSSA ROA, P. Construccion de perfiles psicograficos de profesores de educación básica de escuelas municipales de Arica. Estud. pedagóg., [S.1.], v. 35, n. 1, p.13-26, 2009. Disponible en: $<$ http://www.scielo.cl/scielo.php?pid=S0718-07052009000100001\&script=sci arttext>. Consultado en 22 mayo 2011.

CUADRA-PERALTA,A;FIGUEROA,L.YPLAZA, C. Desarrollo de habilidades sociales y de liderazgo en los mandos medios de la Ilustre Municipalidad de Arica. A través de la implementación de un programa de entrenamiento. Tesis para optar al Título de psicólogo (Cuadra profesor guía). Universidad de Tarapacá. AricaChile, 2002.

CUADRA, H.; FLORENZANO, R. El bienestar subjetivo: hacia una psicología positiva. Revista de Psicología de la Universidad de Chile, [S.1.], v. 12, n. 1, p.83-96, 2003.

DÍAZ MORALES, J.; SÁNCHEZ-LOPEZ, M. Relevancia de los estilos de personalidad y las metas personales en la predicción de la satisfacción vital. Anales de psicología, [S.1.], v. 17, n. 2, p.151-158, 2001. 
Alejandro Adrian Cuadra-Peralta; Leslie Katherine Fuentes-Soto; Daniela Madueño-Soza;

Constanza Beatriz Veloso-Besio; Yerka Bustos Meneses

DIENER, E.; SELIGMAN, M. Beyond money: toward an economy of wellbeing. Psychological Science, [S.1.], v. 5, n. 47, p. 1-31, 2004.

DIENER, E.; OISHI, S.; LUCAS, R. Personality, culture and subjective wellbeing: emotional and cognitive evaluations of life. Annual Review of Psychology, [S.1.], v. 54, n. 1, p. 403-425, 2003.

DINHAM, D; SCOTT, C. Moving into the third, outer domain of teacher satisfaction. Journal of Educational Administration, [S.1.], v. 38, n. 4, p. 379396, 2000.

EMMONS, R.; McCUlLOUGH, M. Counting blessings v/s burdens: an experimental investigation of gratitude and subjective well-being in daily life. Journal of Personality and Social Psychology, [S.1.], v. 84, n. 2, p. 377-389, 2003.

FERNÁNDEZ-RÍOS, M. (Org.; Dir.). Diccionario de Recursos Humanos. Madrid: Días de Santos, 1999.

FREDRICKSON, B. L. The role of positive emotions in positive psychology: the broadening and bulid theory of positive emotions. American Psychology, [S.1.], v. 56, n. 3, p. 218-226, Mar. 2001.

FREIBERG, H. G. School climate: measuring, improving and sustaining healthy environments. Londres: Falmer, 1999.

GANZACH, Y. Intelligence, education, and facets of job satisfaction. Work \& Occupations, [S.1.], v. 30, n. 1, p. 97-123, 2003.

GARCÍA-VINIEGRAS, C.; GONZÁLEZ, I. La categoría bienestar psicológico, su relación con otras categorías sociales. Revista Cubana de Medicina Integral, [S.1.], v. 16, n. 6, p. 586-592, 2000.

JESÚS CAVA M.; MUSITU, G. Autoestima y percepción del clima escolar en niños con problemas de integración social en el aula. Revista de Psicología General, [S.1.], v. 54, n. 2, p. 297-311, 2001.

KLEIN, K. et al. Is everyone in agreement? An exploration of within-group agreement in employee perceptions of the work environment. Journal of Applied Psychology, [S.1.], v. 86, n. 1, p. 3-16, 2001.

LACUNZA, A. B; GONZÁLEZ, N. C. Las habilidades sociales en niños preescolares en contextos de pobreza. Cienc. psicol., Montevideo, v. 3, n. 1, p. 5766, maio 2009. Disponible en: <http://pepsic.bvsalud.org/scielo.php?script=sci arttext\&pid $=\mathrm{S} 1688-40942009000100006 \& \operatorname{lng}=\mathrm{pt} \& \mathrm{nrm}=\mathrm{iso}>$. Consultado en $2 \overline{2}$ mayo 2011. 
LIBERALESSO, A. Bienestar subjetivo en la vida adulta y la vejez: hacia una psicología positiva en América Latina. Revista Latinoamericana de Psicología, [S.1.], v. 34, n. 1-2, p. 55-74, 2002.

LYUBOMIRSKY, S.; KING, L. Y.; DIENER, E. The benefits of frequent positive affect: does happiness lead to success?. Psychological Bulletin, [S.1.], v. 131, n. 6, p.803-855, 2005.

MAÑAS, M., et al. La satisfacción y el bienestar psicológico como antecedentes del compromiso organizacional. Psicothema, [S.l.], v. 19, n. 3, p. 395-400, 2007.

MARTÍN BRIS, M.. Clima de trabajo y participación en la organización y funcionamiento de los centros de educación. Madrid: Universidad de AlcaláMEC. 1999.

MARTÍNEZ, P. Perspectiva temporal futura y satisfacción con la vida a lo largo del ciclo vital. Revista de Psicología, [S.1.], v. 22, n. 2, p. 215-252, 2004.

McCUllough, M. E.; PARGAMENT, K. I.; THORESEN, C. E. (Ed.). The frontiers of forgiveness: seven directions for psychological research and practice. In: . Forgiveness: theory, research, and practice. New York: Guilford, 2000. p. 1-14.

METLER, C. Job satisfaction and perception of motivation among middle and high school teachers. American Secondary Education, [S.1.], v. 31, n. 1, p.43-53, 2002.

MOLINA DE COLMENARES, N.; PÉREZ DE MALDONADO, I. El clima de relaciones interpersonales en el aula un caso de estudio. Paradigma, [S.1.], v. 27, n. 2, p. 193-219, 2006.

MOYANO, E.; RAMOS, N. Bienestar subjetivo: midiendo satisfacción vital, felicidad y salud en población chilena de la Región Maule. Universum, [S.1.], v. 22, n. 2. p. 177-193, 2007.

PEARSON, L.; MOARNAW, W. The relationship between teacher autonomy and stress, work satisfaction, empowerment, and professionalism. Educational Research Quarterly, [S.1.], v. 29, n. 1, p. 37-53, 2005.

PÉREZ, T. Efectos de la aplicación de un programa de educación para la convivencia sobre el clima social del aula en un curso de $2^{\circ}$ de ESO. Revista de Educación, [S.1.], n. 343, p. 503-522, 2007.

PRELIP, M. Job satisfaction in health education and the value added of 
Alejandro Adrian Cuadra-Peralta; Leslie Katherine Fuentes-Soto; Daniela Madueño-Soza;

Constanza Beatriz Veloso-Besio; Yerka Bustos Meneses

credentialing. American Journal of Health Education, [S.1.], v. 32, n. 1, p. 26-30, 2002.

ROMERO, G.; CABALLERO, A. Convivencia, clima de aula y filosofía para niños. Revista Electrónica Interuniversitaria de Formación del Profesorado, [S.1.], v. 11, n. 3, p. 23-31, 2008.

SCHAUFELI, W. B.; SALANOVA, M. La evaluación de riesgos psicosociales en el trabajo. Revista de Prevención, Trabajo v Salud, [S.1.], v. 20, p. 4 -9, 2002.

SELIGMAN, M; CSIKSZENTMIHALYI, M. Positive psychology: an introduction. American Psychologist, [S.1.], v. 55, n. 1, p. 5-14, Jan. 2000.

SELIGMAN, M. Positive Psychology, positive prevention and positive therapy. In: SNYDER, C.; LÓPEZ, S. (Ed.). Handbook of positive psychology. New York, NY: Oxford University Press. 2002. p. 3-12.

SELIGMAN, M. Foreword: the past and future of Positive Psychology. In: KEYES, C. L. M.; HAIDT, J. (Ed.). Flourishing: Positive Psychology and the Life Well-Lived. Washington, DC: American Psychological Association, 2003. p. xi-xx.

STEMPIEN, L.; LOEB, R. Differences in job satisfaction between general education and special education teachers. Remedial \& Special Education, [S.1.], v. 23, n. 5 , p. $258-268,2002$.

TAPIA, M. La solidaridad como pedagogía. Buenos aires: Ciudad Nueva. 2004.

TORRES, J. Educación en tiempos de neoliberalismo. Madrid: Morata. 2001.

TRIANES, M. V. Eficacia de un programa de intervención para la mejora del clima escolar: algunos resultados. Revista Electrónica de Investigación Psicoeducativa, [S.1.], v. 4, n. 2, p. 353-370, 2006.

UNESCO. Estudio cualitativo de escuelas con resultados destacables en siete países Latinoamericanos. Santiago: Oficina Regional de Educación para América Latina y el Caribe, 2002.

VERAPOSECK, B. Resistiry rehacerse: una reconceptualización de la experiencia traumática desde la psicología positiva. Revista de Psicología Positiva, [S.1.], v. $1,2004$.

WARR; COOK; WALL. Scales for the measurement of some work attitudes and aspects of psychological well-being. Journal of Occupational Psychology, [S.1.], v. 52, n. 2, p.129-148, 1979. 
WATSON, S. Activities to support good social skills in the classroom. Retrieved January, v. 22, 2007.

ZABALZA, M. A. El clima: conceptos, tipos, influencias del clima e intervención sobre el mismo. In: DOMINGUEZ, G; MESANZA, J. (Coord.). Manual de organización e instituciones educativas. Madrid: Escuela Española. 1996. p. 263301.

Recebido em: 23 de maio de 2011

Aceito em: 19 de março de 2012 
